Results The density of SIGLEC1 molecules on the surface of monocytes based on two visits, correlated with the SLEDAI (128 determinations, betaST $0.22, \mathrm{p}<0.012$ ), but not with the C3 (108 determinations, betaST 0.03, p=0,80), the C4 (106 determinations, betaST $-0.06, \mathrm{p}<0.58)$ and the anti-dsDNAantibodies (104 determinations, betaST 0.06, $\mathrm{p}=0.61)$. SIGLEC1 is a more change-sensitive biomarker than the conventional laboratory parameters C3, C4 and ds-DNA-Ab. Patients with an increase in SIGLEC1 of >1120 molecules/ monocyte between two visits show a higher probability $(\mathrm{OR}=7.0, \mathrm{p}<0.001)$ of minimal clinical worsening (SLEDAI more/equal 2 points). Patients who show a decrease $>2902$ SIGLEC1 molecules/monocyte have a higher chance $(\mathrm{OR}=6.3$, $\mathrm{p}=0.004$ ) to have a clinical improvement (SLEDAI more/equal 2 points).

Conclusion This prospective cohort study showed for the first time the significant relationship between the routinely measured interferon biomarker SIGLEC1 and the disease activity in paediatric SLE patients. Thus, SIGLEC1 represents a potential marker for activity monitoring in this disease.

\section{PS1:13 SYSTEMIC LUPUS ERITHEMATOSUS WITH POSITIVE ANTICENTROMERE ANTIBODIES - A DIFFERENT CLINICAL SUBGROUP?}

'J Caetano, ${ }^{1} \mathrm{C}$ Favas, ${ }^{1,2} \mathrm{M}$ Amaral, ${ }^{1} \mathrm{~S}$ Oliveira, ${ }^{1,2} \mathrm{JD}$ Alves. ${ }^{1}$ Systemic Autoimmune Diseases Unit, Department of Medicine IV, Fernando Fonseca Hospital, Amadora, Portugal; ${ }^{2}$ CEDOC/ NOVA Medical School, Lisbon, Portugal

\subsection{6/lupus-2018-abstract.61}

Introduction Anticentromere antibodies (ACA) are one of the most specific systemic sclerosis (SSc)-related antibodies. The presence of ACA has also been identified in other autoimmune diseases, mainly in SSc overlap syndromes with Sjogren syndrome $(\mathrm{SjS})$, primary biliary cirrhosis and rarely in patients with systemic lupus erythematosus (SLE).

Purpose To evaluate the prevalence and clinical significance of ACA in a cohort of SLE patients.

Methods Retrospective analysis of all ACA positive SLE patients (ACA +SLE), from a cohort of 270 consecutive SLE patients fulfilling the 2012 SLICC Criteria and/or 1997 ACR Criteria, of a single referral centre, between 2010-2016. Comparative analysis was made with a representative group of 63 consecutive SLE patients without ACA (ACA-veSLE). Data were obtained by medical records review.

Results From 270 SLE patients, 10 (3.7\%) were ACA+. All ACA + SLE patients were female. The age at the time of diagnosis was not different between the groups (40.9 \pm 16.6 years ACA +SLE vs 37.8 \pm 16.2 years ACA-veSLE), but ACA +SLE patients had longer disease duration (15.2 \pm 17.3 years vs 9.5 \pm 8.8 years, $\mathrm{p}=0.002$, respectively). ACA + SLE patients had significantly more Raynaud's phenomenon (RP) $(p=0.028)$, but none had a capillaroscopy SSc pattern. Sicca symptoms were also more frequent in ACA +SLE $(p=0.013)$, with only 1 patient with a positive anti-SSA antibody. None of these patients fulfilled criteria for SjS.

Prevalence of arthritis, oral ulcers, alopecia, cutaneous lupus, serositis, neurologic, renal and hematologic involvement was not significantly different between the two groups. Hypocomplementemia at any time of the disease course was more frequent in ACA-veSLE $(p=0.016)$.
Antiphospholipid antibodies were less frequently positive in ACA+SLE patients ( $20 \%$ vs $46 \%, p=0.1)$, and none fulfilled criteria for antiphospholipid syndrome (APS) (21\% of ACAveSLE patients with APS).

Apart from RP, SSc-associated clinical characteristics (skin thickening, digital ulcers, telangiectasia, pulmonary arterial hypertension, interstitial lung disease, gastroesophageal reflux and calcinosis) were not present in any of the ACA+SLE patients.

Conclusions ACA +SLE patients do not constitute a different clinical subgroup regarding organ involvement, but can associate with a lower probability of concomitant APS. Moreover, although highly specific of SSc, ACA can be identified in SLE patients without SSc overlap, and should not hamper the diagnosis of SLE.

\section{PS1:14 TRPV1 RECEPTOR ACTIVITY IN LUPUS}

M Sahebari, SZ Mirfeizi, I Salimi. Rheumatic Diseases Research Centre, Mashhad, Iran

10.1136/lupus-2018-abstract.62

Objective: The etiopathogenesis of lupus is still not fully understood. According to cumulative data, immune, environmental, genetic and neruendocrine factors interact to develop SLE. TRPV1 receptors on neuronal cells are parts of an important inflammatory pathway in autoimmunity. The role of TRPV1 receptors as a calcium dependent channel receptor in pro inflammatory cell reactions is under investigation. Capsaicin an extract of capsicum and oleoresin capsicum has high affinity to TRPV1 receptors. Substance-P discharge from TRPV1 receptors lead to pain relief. The same mechanism may be responsible for resolving inflammation. It is a hypothesis that the skin reaction to Capsaicin is an estimation activity of TRPV1 receptors in autoimmune diseases.

Method 29 female lupus patients and 33 healthy age and sex match volunteers who passed the inclusion criteria of the disease and the study were enrolled. For each participant, a $1 \times$ $1 \mathrm{~cm} 2$ blotting paper imbrued by $0.1 \mathrm{ml}$ of the capsaicin solution $(0.075 \%)$ from Sigma Company was put on the volar forearm and covered by a plastic band, to prevent evaporation. The test was then carried out which consisted of time to tingling, induration area $(\mathrm{cm} 2)$, and redness area $(\mathrm{cm} 2)$; measured after $15 \mathrm{~min}$.

Results The mean age of patients was 30 (25.5-41.5) and controls was $35(28-48.5)$ years $(p=0.09, z=-1.6)$. Tingling sensation was sensed by $22(75.9 \%)$ of patients and $12(36.4 \%)$ of controls $(\mathrm{p}=0.01, \mathrm{x} 2=13)$. Redness was observed in 18 $(62.11 \%)$ of patients and $8(24 \%)(\mathrm{p}<0.01, \mathrm{x} 2=9.07)$. Time to tingling in SLE and controls was 6.5 (4.75-9) and 3 (24) $\min (p=0.02, z=-2.39)$. Redness area after $15 \mathrm{~min}$, in SLE and controls were $8.05(0-12) \mathrm{cm}^{2}$ and $0(0-.24) \mathrm{cm}^{2}$, respectively $(\mathrm{p}<0.01, \mathrm{z}=-3.38)$. In lupus group, induration area was $1.5(0-3.25) \mathrm{cm} 2$ and in controls it was $0(0-0)$, $(\mathrm{p}<0.001, \mathrm{z}=-3.38)$.

Conclusion This study suggested that skin reaction to capsaicin in lupus patients is statistically significant stronger than normal individuals. It may stem from more substance-P release from nerve endings or more active TRPV1 receptors in lupus. Studies on TRPV1 pathway in lupus are limited. We suggest that this pathway plays some role in lupus pathogenesis and more researches on this purpose should be considered. 